distribution curves crossed each other. Our clinical cut-point was chosen to differentiate best between subjects with subjective complaints and AD-dementia and was $550 \mathrm{pg} / \mathrm{ml}$. Results: Visual inspection suggested a trimodal distribution (figure). Mixture modeling estimated a bimodal distribution and yielded a cut-point at $639 \mathrm{pg} / \mathrm{ml}$. The best cut-point was lower in subjects younger than 70 years $(607 \mathrm{pg} / \mathrm{ml})$ than in subjects older than 70 years (713 pg/ml). Cut-points were comparable in subjects with subjective complaints $(649 \mathrm{pg} / \mathrm{ml})$, MCI $(664 \mathrm{pg} / \mathrm{ml})$ and dementia $(638 \mathrm{pg} / \mathrm{ml})$. Cut-points did not differ with APOE genotype $(644 \mathrm{pg} / \mathrm{ml}$ in both APOE-e4 carriers and non-carriers). $81 \%$ of the subjects with AD had CSF abeta 1-42 concentrations below the cut-point based on clinical diagnosis and $91 \%$ below the $639 \mathrm{pg} / \mathrm{ml}$ cut-point. Conclusions: Cut-points for abnormal CSF abeta1-42 based on unbiased mixture modeling are higher than those based on clinical diagnosis. This suggests that diagnosis-based cut-points may underestimate the prevalence of abnormal CSF abeta 1-42. Cut-points were dependent on age but independent of degree of cognitive impairment and APOE genotype. Longitudinal studies are needed to determine the outcome of subjects without a clinical diagnosis of AD that had abeta1-42 levels between 550 and $639 \mathrm{pg} / \mathrm{ml}$.

\section{O1-09-04 BASELINE OR PROGRESSION? BIOMARKERS PREDICTIVE OF MEMORY DECLINE}

Hiroko Dodge $^{1}$, Jian Zhau $^{2}$, Danielle Harvey ${ }^{3}$, Lisa Silbert ${ }^{4}$, Jeffrey Kaye ${ }^{4}$, Roger Albin ${ }^{2},{ }^{1}$ Oregon Health \& Science University/University of Michigan Health System, Portland, Oregon, United States; ${ }^{2}$ University of Michigan, Ann Arbor, Michigan, United States; ${ }^{3}$ University of California, Davis,
Davis, California, United States; ${ }^{4}$ Oregon Health \& Science University, Portland, Oregon, United States. Contact e-mail: hdodge@med.umich.edu

Background: It is not known which component of commonly employed Alzheimer's disease (AD) biomarkers -baseline value or progression-is a better predictor of cognitive decline. We examined how much of individual differences in cognitive decline (variability in longitudinal slope) measured by neuropsychological tests can be explained by changes/progression of biomarkers as opposed to their baseline values at each $\operatorname{cog}$ nitive stage (normal, MCI, mild AD). Methods: 526 subjects in the Alzheimer's Disease Neuroimaging Initiative (ADNI) with valid data in all of our variables of interest were used in this study. The primary clinical outcome is the cognitive composite score tapping the memory domain. Baseline values and progression in the following biomarkers were examined in their association with trajectory of the cognitive outcome: MRI total brain, hippocampal, ventricular, WMH volumes, ROI cortical thickness (medial and inferior temporal thickness), FDG-PET summary score $(\mathrm{n}=260)$ and CSF p-tau, t-tau and abeta42 $(\mathrm{n}=271)$. First, individual-specific slope (i.e., random component) of the longitudinal trajectory of each biomarker was estimated using mixed effects models, controlling for age, sex, education, practice effects, apoe 4 allele and changes in diagnosis. Then these estimates and observed baseline values were used as predictors of cognitive decline using mixed effects models. Variability in cognitive decline (i.e., individual differences in slopes) explained by the subject-specific baseline biomarker values was compared with that explained by the progression. Results: Even among the normal subjects where cognitive decline is minimal, progression in FDG-PET (but not baseline) explained the variability in memory decline. Also progression explained variability in

Table 1

Proportion of memory decline (variability in longitudinal slope) explained by each biomarker: baseline and change values

\begin{tabular}{|c|c|c|c|c|c|c|}
\hline $\begin{array}{l}\text { Biomaker_bl: baseline } \\
\text { values_progression: } \\
\text { changes }\end{array}$ & \multicolumn{2}{|l|}{ Normal Group } & \multicolumn{2}{|l|}{ Among MCI* } & \multicolumn{2}{|l|}{ Among AD } \\
\hline ttau_bl & $-7.00 \%$ & 0.02 & $-0.40 \%$ & -0.01 & $-7.90 \%$ & -0.19 \\
\hline ttau_progression & $-7.80 \%$ & -0.02 & $-1.50 \%$ & -0.02 & $-17.80 \%$ & -0.08 \\
\hline Abeta $_{42 \_b l}$ & $-5.90 \%$ & 0.01 & $4.40 \%$ & 0.08 & $-8.60 \%$ & -0.05 \\
\hline FDG-PET_progression & $2.80 \%$ & 0.05 & $14.60 \%$ & 0.05 & $39.40 \%$ & 0.12 \\
\hline Wmh/icv_bl & $-4.20 \%$ & 0.04 & $0.70 \%$ & -0.02 & $-4.80 \%$ & 0.09 \\
\hline $\mathrm{Wmh} /$ icv_progression & $-2.20 \%$ & -0.04 & $-0.20 \%$ & 0.01 & $2.80 \%$ & 0.11 \\
\hline Hpcv/icv_bl & $-0.40 \%$ & 0.04 & $9.60 \%$ & 0.06 & $3.40 \%$ & -0.14 \\
\hline Hpcv/icv_progression & $-4.40 \%$ & 0 & $15.60 \%$ & 0.06 & $2.60 \%$ & 0.12 \\
\hline Ventricles/icv_bl & $-0.70 \%$ & -0.01 & $8.60 \%$ & -0.07 & $-0.30 \%$ & 0 \\
\hline mtthickness/icv_bl & $-3.60 \%$ & -0.01 & $5.60 \%$ & 0.08 & $7.20 \%$ & 0.09 \\
\hline $\begin{array}{l}\text { mtthickness/ } \\
\text { icv_progression }\end{array}$ & $-1.10 \%$ & 0.03 & $22.50 \%$ & 0.08 & $51.40 \%$ & 0.15 \\
\hline itthickness/icv_bl & $-2.40 \%$ & -0.01 & $4.80 \%$ & 0.08 & $8.80 \%$ & 0.05 \\
\hline $\begin{array}{l}\text { itthickness/ } \\
\text { icv_progression }\end{array}$ & $1.60 \%$ & 0.03 & $28.30 \%$ & 0.09 & $73.00 \%$ & 0.19 \\
\hline
\end{tabular}

Itthickness: inferior temporal thickness

Tpthickness: temporal pole thickness

Brain volumes and cortical thickness were divided by intracranial volume. Controlling for age at baseline, sex, education, apoe 4 allele (at least one vs. none) and practice effects (an indicator of before 6 months assessment vs. at/after 6 months assessment for memory composite outcome: 6 months was used as a cutoff based on the visual inspection of trajectory and the improvement in model fit). *: to capture the change in diagnosis from MCI to AD during the follow-up, an indicator variable (before MCI coded as 0 , after MCI coded as 1) was included as a control variable to capture the shift in slopes in cognitive decline.

The table can be read as follows: For example, among MCI subjects, one standard deviation larger expansion in icv-adjusted ventricular volume (ventricles/ icv_progression) is associated with 0.08 further decline in memory scores each year (slope effect: -0.08 ) and the progression explains $27.6 \%$ of variability in cognitive decline, while baseline explains only $8.6 \%$ of variability in cognitive decline. 
memory decline more than the baseline values in most biomarkers; the proportion of variability explained ranged from $14.6 \%$ (changes in FDT-PET) to $28.3 \%$ (changes in inferior temporal thickness) among the MCI subjects. Among AD subjects, an even higher proportion was explained by the progression: 39.4\% (changes in FDG-PET), 51.4\% (changes in medial temporal thickness), $68.9 \%$ (ventricular expansion), $73.0 \%$ (changes in inferior temporal thickness). Conclusions: Progression in biomarkers is more important than baseline values in most biomarkers in predicting cognitive decline. This has important implications for clinical trials targeted to modify $\mathrm{AD}$ biomarkers, as well as for prognosis and prediction of clinical outcomes.

\section{O1-09-05 FURTHER EVALUATION OF THE INDICATIONS FOR THE RATIONAL USE OF CSF IN THE DIAGNOSTICS OF ALZHEIMER'S AND RELATED DISORDERS}

Gorazd Bernard Stokin ${ }^{1}$, Marko Privošnik ${ }^{2},{ }^{1}$ University Psychiatric Hospital, Ljubljana, Slovenia; ${ }^{2}$ Faculty of Computer and Information Science, University of Ljubljana, Ljubljana, Slovenia.

Contacte-mail: gbstokin@alumni.ucsd.edu

Background: Advancements in our understanding of the biology of Alzheimer's and related diseases culminated in the proposal of novel criteria for the clinical diagnosis of Alzheimer's disease. This research criteria reject the previous probabilistic diagnostic approach in favor of a confirmatory one. More specifically, they propose demonstrating some characteristic of Alzheimer's disease including amyloidogenesis and/or neurodegeneration in addition to the cognitive decline characteristic of Alzheimer's disease. It remains currently unclear what methods to demonstrate amyloidogenesis and/or neurodegeneration should be used at different points in the diagnostics of Alzheimer's and related diseases. Methods: We are prospectively recording all patients in our memory clinic that require cerebrospinal fluid analyses in the process of diagnosing the cause of their behavioral changes and cognitive decline. All our patients undergod behavioral and cognitive testing, neuropsychiatric exam, MRI including manual and semi-automated volumetry and when required cerebrospinal fluid analyses including basic cerebrospinal parameters, reibergrams, testing for infectious diseases, amyloid 1-42, tau, phospho-tau, neuron specific enolase, S100 and several markers of inflammation. Results: Here we extend our findings presented last year in Vancouver by increasing the number of patients, by increasing in particular the number of healthy control subjects thus modifying further our cut-off values. Importantly several indications for cerebrospinal fluid analyses when diagnosing Alzheimer's and related disorders appear to emerge. Cerebrospinal fluid analyses is most useful when either cognitive profile in not typical of a specific Alzheimer's or related disorder or when MRI analyses fails to demonstrate neurodegenerative changes in the appropriate anatomical regions. In addition, the use of inflammatory markers might turn out to be of relevance in assessing some of the causes and possibly the rate of progression of neurodegeneration. Conclusions: Our data suggest that cerebrospinal fluid analyses should be performed primarily in cases when cognitive decline and/or MRI imaging fail to meet the requirements proposed in novel research clinical diagnostic criteria for Alzheimer's and related diseases.

\section{O1-09-06 THE 'CSF ALZHEIMER'S PROFILE': EASILY SAID,} BUT WHAT DO WE MEAN?

Flora Duits ${ }^{1}$, Charlotte Teunissen ${ }^{1}$, Femke Bouwman ${ }^{1}$, Pieter Jelle Visser ${ }^{1}$, Niklas Mattsson $^{2}$, Henrik Zetterberg ${ }^{3}$, Kaj Blennow ${ }^{3}$, Oskar Hansson ${ }^{4}$, Lennart Minthon ${ }^{5}$, Niels Andreasen ${ }^{6}$, Jan Marcusson ${ }^{7}$, Anders Wallin ${ }^{8}$, Marcel Olde Rikkert ${ }^{9}$, Magda Tsolaki ${ }^{10}$, Lucilla Parnetti ${ }^{11}$, SannaKaisa Herukka ${ }^{12}$, Harald Hampel ${ }^{13}$, Mony De Leon ${ }^{14}$, Johannes Schröder ${ }^{15}$, Dag Aarsland ${ }^{16}$, Marinus A. Blankenstein ${ }^{1}$, Philip Scheltens ${ }^{1}$, Wiesje Van der Flier ${ }^{1},{ }^{1} V U$ University Medical Center, Amsterdam, Netherlands; ${ }^{2}$ University of California, San Francisco, San Francisco, California, United States and University of Gothenburg, Sweden, Mölndal, Sweden;

${ }^{3}$ Sahlgrenska Academy, University of Gothenburg, Mölndal, Sweden;

${ }^{4}$ Depatment of Neurology, Skåne University Hospital, Malmö, Sweden;
${ }^{5}$ Department of Clinical Sciences, Malmö, Sweden; ${ }^{6}$ Karolinska Institutet, Stockholm, Sweden; ${ }^{7}$ Department of Geriatric Medicine, Linköping, Sweden; ${ }^{8}$ University of Gothenburg, Mölndal, Sweden; ${ }^{9}$ University Medical Center Nijmegen, Nijmegen, Netherlands; ${ }^{10}$ Aristotle University of Thessaloniki, Thessaloniki, Greece; ${ }^{11}$ Clinica Neurologica, Perugia, Italy; ${ }^{12}$ Clinica Neurologica, Kuopio, Finland; ${ }^{13}$ University of Frankfurt,

Frankfurt am Main, Germany; ${ }^{14}$ NYU, New York, New York, United States; ${ }^{15}$ University of Heidelberg, Heidelberg, Germany; ${ }^{16}$ Stavanger Hospital, Stavanger, Norway. Contact e-mail: f.duits@vumc.nl

Background: Cerebrospinal fluid (CSF) amyloid $\beta$ 1-42 (A $\beta 42)$, total tau and phosphorylated tau at threonine 181 (ptau-181) have been described extensively as biomarkers for Alzheimer pathophysiology, but there is still no consensus on how to optimally combine them or how to implement them in clinical practice. With $t$ his study we aimed to identify the most useful definition of the CSF 'Alzheimer profile'. Methods: CSF biomarkers were analyzed using ELISA in 1385 patients from our memory clinic based Amsterdam Dementia Cohort, with subjective memory complaints (controls), mild cognitive impairment (MCI), AD or other dementias. We constructed $\mathrm{AD}$ profiles using ratios of tau/A $\beta 42$ and ptau-181/A $\beta 42$, combinations of dichotomized $\mathrm{A} \beta 42$, tau and ptau-181, and four different regression formulas (previously published by Hulstaert, Mulder, Mattsson and Schoonenboom). We calculated Receiver operating characteristic (ROC) curves and test characteristics for $\mathrm{AD}$ versus controls and versus other dementia patients. We used Cox proportional hazards models to assess the predictive value of all combinations for conversion to AD in MCI patients. Finally, we validated the results in a large independent, multicenter cohort. Results: Ratios and formulas performed better than individual biomarkers and combinations of dichotomized biomarkers to discriminate groups. Based on the sensitivity values of the best performing formulas, we chose cut-offs of 0.52 for tau/A $\beta 42$ and 0.08 for ptau-181/A $\beta 42$. Simple ratios were as accurate as regression formulas; sensitivity for $\mathrm{AD}$ was 91$93 \%$, specificity for controls $81-84 \%$, and specificity for other dementias $63-74 \%$. Ratios were also better predictors of conversion from MCI to $\mathrm{AD}$ than combinations of dichotomized biomarkers and performed comparable to regression formulas, with hazard ratios of 8 to 10 . Results of the validation cohort were remarkably similar, especially for the ratio of tau/A $\beta 42$, but also for the formulas of Mulder and Hulstaert. Based on these results, a simple ratio of tau/A $\beta 42>0.52$ constitutes the optimal CSF Alzheimer profile. Conclusions: Based on the principle of parsimony, we recommend using a simple ratio to combine CSF biomarkers. Based on these data, the optimal CSF Alzheimer profile exists whenever tau is more than half the value of $\mathrm{A} \beta 42$, independent of the clinical question at hand.

\section{ORAL SESSIONS: O1-10 \\ DIAGNOSIS AND PROGNOSIS: IMAGING PREDICTORS OF COGNITION}

\section{1-10-01 PREDICTING ALZHEIMER'S-RELATED COGNITION WITH CORTICAL THICKNESS CORRELATIONS: A GLMNET APPROACH}

Andrew Reid, Alan Evans, Montreal Neurological Institute, Montreal, Quebec, Canada.Contact e-mail: areid@bic.mni.mcgill.ca

Background: Alzheimer's disease (AD) has been proposed to be primarily a "disconnection syndrome", whereby the progression of neurodegeneration propagates along cortical networks. Previously, we have introduced methods by which cognitive performance can be predicted from groupwise correlations in cortical thickness, derived from T1-weighted MRI. Here, we extend this basic approach using GLMNET, which allows all network edges to be analyzed in a single multivariate regression model, to yield a small set of edges which best predict the individual behavioural outcome. Methods: Cortical thickness estimates were obtained from T1-weighted images obtained from the ADNI-1 cohort. For each pair of regions (ROIs), a linear model was fitted for Normal Control (NC) subjects only. Residual error was computed from this model for all subjects. Using GLMNET, the resulting residuals were regressed against cognitive performance scores in a general linear model (GLM) including residuals from all pairs of ROIs i 